\title{
EpiDope: A Deep neural network for linear B-cell epitope prediction
}

\author{
Maximilian Collatz ${ }^{1, *}$, Florian Mock ${ }^{1, *}$, Martin Hölzer ${ }^{1,5}$, Emanuel Barth ${ }^{1,2}$, Konrad Sachse ${ }^{1}$, \\ and Manja Marz $z^{1,2,4,5}$ \\ ${ }^{1}$ RNA Bioinformatics and High-Throughput Analysis, Friedrich Schiller University Jena, Germany \\ ${ }^{2}$ Bioinformatics Core Facility, Friedrich Schiller University Jena, Jena, Germany \\ ${ }^{4}$ FLI Leibniz Institute for Age Research, Jena, Germany \\ ${ }^{5}$ European Virus Bioinformatics Center, Jena, Germany \\ * contributed equally to this work
}

\begin{abstract}
By binding to specific structures on antigenic proteins, the so-called epitopes, B-cell antibodies can neutralize pathogens. The identification of B-cell epitopes is of great value for the development of specific serodiagnostic assays and the optimization of medical therapy. However, identifying diagnostically or therapeutically relevant epitopes is a challenging task that usually involves extensive laboratory work. In this study, we show that the time, cost and labor-intensive process of epitope detection in the lab can be significantly shortened by using in silico prediction. Here we present EpiDope, a python tool which uses a deep neural network to detect B-cell epitope regions on individual protein sequences (github.com/ mcollatz/EpiDope). With an area under the curve (AUC) between $0.67 \pm 0.07$ in the ROC curve, EpiDope exceeds all other currently used B-cell prediction tools. Moreover, for AUC10\% (AUC for a false-positive rate $<0.1$ ), EpiDope improves the prediction accuracy in comparison to other state-of-the-art methods. Our software is shown to reliably predict linear B-cell epitopes of a given protein sequence, thus contributing to a significant reduction of laboratory experiments and costs required for the conventional approach.
\end{abstract}

\section{Introduction}

The public health system is highly dependent on the use of vaccines to protect the population from a range of dangerous infectious diseases. Through decades of systematic vaccination, diseases like measles, mumps, rubella, pertussis, poliomyelitis, diphtheria, tetanus and others have been largely eradicated [1, 2]. Vaccination is also an efficient approach to avoid or reduce prescriptions of antibiotics and, as a consequence, minimize the emergence of ever more multi-resistant strains of microbial pathogens. To assess the degree of protection of vaccination at population level, faster and more efficient serological tools need to be developed. They should be capable of identifying geographical and social heterogeneities in the diversity of population immunity [3, 4]. In addition, it is important to know the status of a patient's immunization in order to avoid unnecessary vaccinations. In cases where this is not or only incom- pletely documented, various tests can be used to determine which specific immunities already exist and which vaccinations are missing. The serological tests currently used, however, are still largely based on ELISA technology and are only able to detect antibodies against a single, specific pathogen [4]. These tests are not only slow and expensive, but usually also use whole cell antigens to detect antibodies, which limits their specificity [5].

B-cell antibodies of the immune system of a host are able to detect certain exposed amino acids and subsequently bind the corresponding antigenic proteins. These bound protein regions are called epitopes and represent the interface between infection and immune response [6, 7]. The antibody part that binds the epitope is called paratope. Epitopes themselves are not intrinsic features of a protein, but rather relational units defined by the interaction with a binding paratope [6]. This relatively vague definition makes it a challenging task to predict epitopes in silico [7, 8, Furthermore, epitopes 
are divided into linear and conformational epitopes, where linear epitopes consist of a contiguous piece of amino acids and conformational epitopes consist of atoms of surface residues that come together by protein folding [6]. In this study, we will focus on the prediction of linear B-cell epitopes.

A frequently used tool to predict linear B-cell epitopes is BepiPred2 [9]. Jespersen et al. state an area under the curve (AUC) of 0.57 for their tool's receiver operating characteristic (ROC) curve. This is on par with other prediction scales provided by the "Immune Epitope Database" IEDB (http://tools.iedb.org/ main/bcell/) and demonstrates the difficulty of in silico epitope identification. Therefore, we developed EpiDope, a tool based on deep neural networks (DNN) to detect epitopic regions in proteins based on their primary amino acid sequence.

DNNs are often used in complex classification problems with limited knowledge about useful features of the objects to be classified. With sufficient data, a DNN can automatically recognize appropriate classification features, making DNNs very suitable for the prediction of linear B-Cell epitopes [10].

We will show that our DNN-based program EpiDope succeeds in identifying linear B-cell epitopes with a ROC AUC of $0.67 \pm 0.07$, which significantly exceeds previous methods. This helps to considerably reduce the number of potential linear epitopes to be validated experimentally and, thus, can accelerate the development of serological assays and immunotherapeutic approaches.

\section{Material and Methods}

\section{Data}

The "IEDB Linear Epitope Dataset" (available at http://www.cbs.dtu.dk/services/BepiPred/ download.php), which was also used for the evaluation of the B-cell epitope prediction tool BepiPred2 [9], served as a training basis for our DNN. It consists of 30,556 protein sequences, in which each sequence contains a marked region, in the following called 'verified regions', that represents an experimentally verified epitope or non-epitope. The subset of epitopes has a average length of 13.99, whereas the subset of non-epitopes has a average length of 13.20 (see Table 1).

\section{Data preparation}

In order to ensure the best possible training basis for the DNN, we pre-processed the dataset in several steps (see Figure 1). First, we merged identical protein sequences while keeping the information about their verified regions, resulting in a reduced dataset containing 3,158 proteins preserving all 30,556 verified regions (Figure 1 A and B). Second, to reduce redundancy by non-identical but highly similar protein sequences, we clustered all sequences with cd-hit [11] using an identity threshold of 0.8 (Figure $1 \mathrm{C}$ ). This resulted in 1,798 protein sequence clusters. From each cluster, only the protein sequence containing the largest number of verified regions was retained, reducing the number of verified regions by $19.46 \%$ to 24610 (see Table 1, Figure 1 D). This reduces the number of very similar and thus overrepresented proteins in the data, as these might bias the training of the DNN.

Table 1: Comparison of the original dataset provided by Jespersen et al. [9], and the redundancy reduced data used as training data.

\begin{tabular}{l|l|l} 
& original & reduced \\
\hline \# verified regions & 30,556 & 24,610 \\
\# epitopes & 11,834 & 8,519 \\
\# non-epitopes & 18,722 & 16,091 \\
\hline median length & 15 & 15 \\
avg. length & 13.50 & 13.27 \\
avg. length epitope & 13.99 & 13.88 \\
avg. length non-epitope & 13.20 & 12.95
\end{tabular}

The clustering step was repeated on the reduced protein sequences using an identity threshold of 0.5 , resulting in 1,378 sequence clusters. These clusters were then used to build the 10-fold cross-validation for the DNN, where the data was divided into ten equally sized subsets according to the number of clusters. This sequence reduction approach was implemented with the following advantage in mind: The DNN training, and test sets share no proteins with a sequence identity of more than 0.5 , ensuring that both sets are as independent as possible from each other, avoiding the DNN from simply memorizing specific epitopes.

\section{Deep neural network architecture}

We compared several DNN architectures, including different ordering, layer types and numbers of nodes. The DNN architecture used in EpiDope consists of two parts (see Figure 22). The first part (Figure 2 A) uses context-sensitive embeddings of 
bioRxiv preprint doi: https://doi.org/10.1101/2020.05.12.090019; this version posted May 25, 2020. The copyright holder for this preprint (which was not certified by peer review) is the author/funder, who has granted bioRxiv a license to display the preprint in perpetuity. It is made available under aCC-BY-NC 4.0 International license.

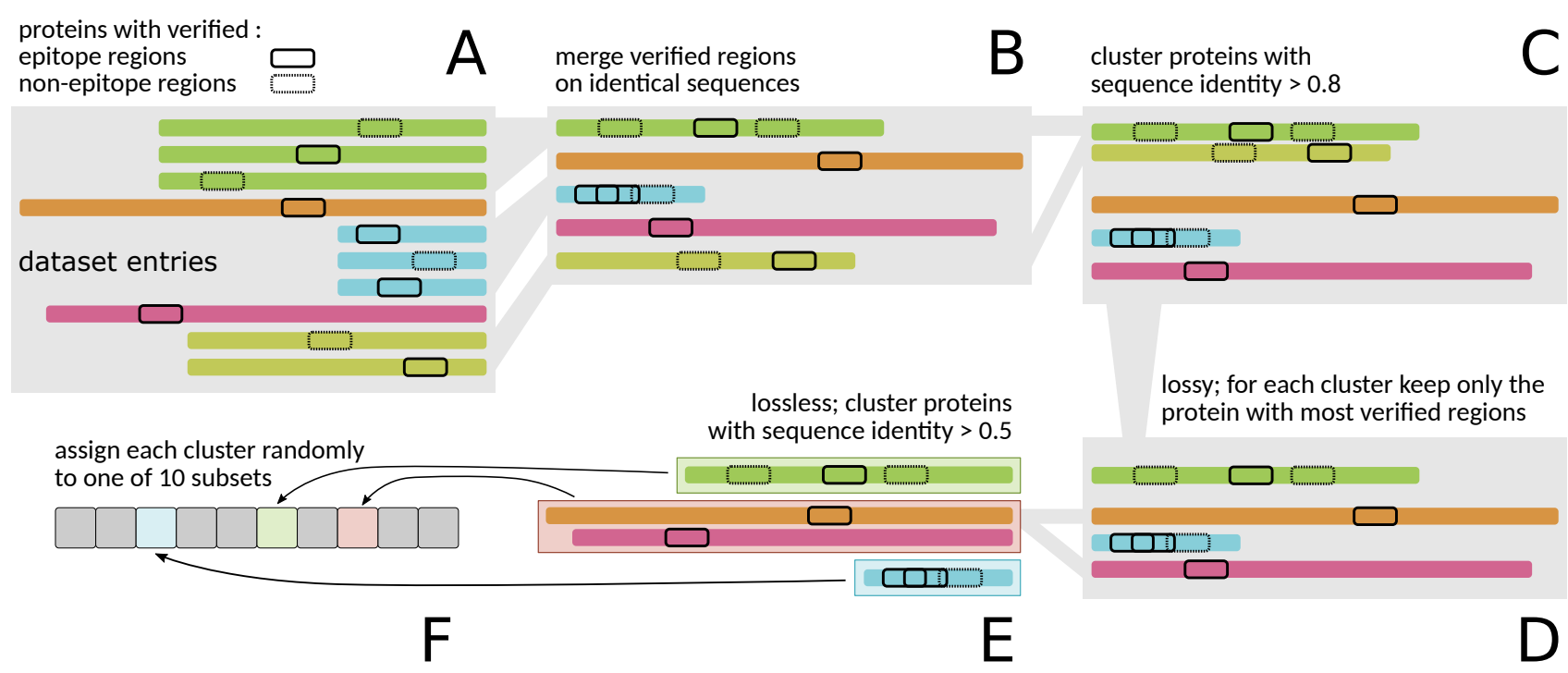

Figure 1: The data preparation to generate the training and validation set. First all identical amino acid sequences from the raw dataset (A) are merged while preserving the verified regions (B). In the next step (C), all remaining sequences are clustered with a sequence identity of 0.8 and higher. For each of the resulting clusters only the sequence with the highest number of verified regions is selected (D). These selected sequences are then clustered again, this time with a sequence identity of 0.5 and higher (E). Each cluster is then assigned to only one of ten different subsets for the 10 -fold cross-validation $(\mathrm{F})$. The data preparation generates training and test data with a low sequence identity of $<0.5$ while limiting the loss of potential sequences.

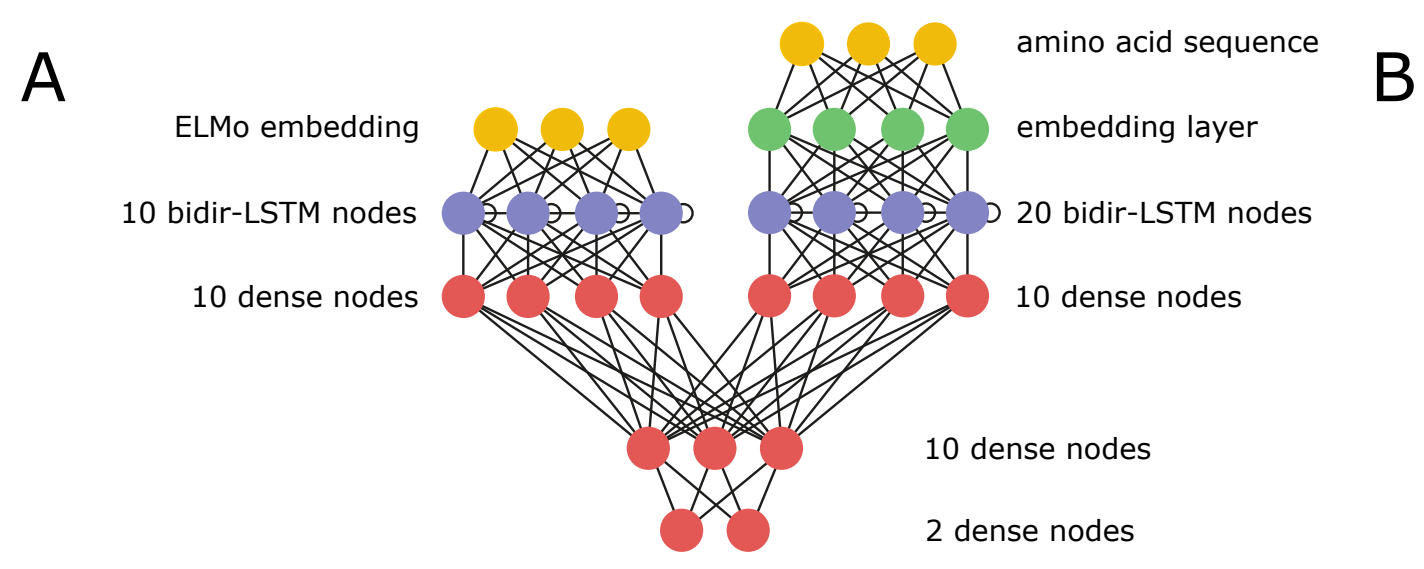

Figure 2: The DNN architecture of EpiDope consists of two parts. The first (A) uses context-sensitive ELMo embeddings for the epitope prediction. These embeddings are previously calculated by an ELMo DNN. The second part (B) uses classic embeddings for prediction. These classic embeddings are not context-sensitive. Both parts are then joined to predict two classes, epitopes and non-epitopes. 
amino acids produced by an ELMo DNN. This DNN was previously trained by Heinzinger et al. to encode various chemical, physical and structural information and was demonstrated to be usable for various high-quality predictions on protein sequences [12]. Each amino acid of a given protein sequence is encoded in a vector of length 1,024 which encodes the chemical, physical and structural information. These embeddings are the input for a bidirectional LSTM layer with $2 \times 5$ nodes [13, followed by a dense layer containing ten nodes.

The second part of our DNN architecture (Figure $2 \mathrm{~B}$ ) encodes each amino acid into a vector of length ten. This embedding is not context-sensitive and is trained together with the rest of the DNN. This embedding layer is connected to a bidirectional LSTM layer with $2 \times 10$ nodes, again followed by a dense layer with ten nodes.

Both dense layers are then connected with an additional dense layer containing ten nodes, which is concluded by the output layer with two nodes representing the two classes, epitope and non-epitope. Note, the number of nodes in this DNN is comparatively low. However, due to the high dimensionality of the context-sensitive embedding (1024 per amino acid), the number of parameters tuned by the DNN is substantial.

\section{Evaluation of epitope prediction approaches}

We compared our tool EpiDope against five frequently tools for linear B-cell epitope prediction from the IEDB (http: //tools.iedb.org/bcell// in their latest version. Namely Bepipred 2.0 [9], Parker Hydrophilicity prediction 14, Chou and Fasman beta turn prediction [15], Emini surface accessibility scale [16, Kolaskar and Tongaonkar antigenicity scale [17] and the independent prediction tool for intrinsically unstructured protein regions IUPred [18].

For each tool, we calculated the corresponding prediction values for the entire protein sequence on each amino acid and sliced out the verified regions. For each of the sliced regions, we calculated the average score as the prediction score to discriminate between epitope and non-epitope. For the antigenicity scale approach we additionally subtracted the mean of the full protein as suggested in the original paper [17.

We compared all the tools using ROC and Precision-Recall curves. For the EpiDope ROC curve, any subset of the 10-fold cross-validation was predicted by the model that did not include this subset in its training data. We calculated one ROC curve per subset. This resulted in ten ROC curves, on which the mean ROC curve was calculated. In the mean ROC curve each of the ten models had an equal influence. The ROC curves of competing tools are calculated on the same data without having to combine multiple predictions, as these tools and scores did not use this data for training.

For the Precision-Recall curve of EpiDope, as with the ROC curve, each subset was predicted by the model that did not use it in the training set. Next, we calculate the Precision-Recall curve on all ten subsets at once rather than calculating ten Precision-Recall curves. Calculating the mean curve from ten Precision-Recall curves could change the balance between the two classes and as such bias the Precision-Recall curve. All other Precision Recall curves were calculated equally.

The ROC curves as well as the Precision-Recall curves were calculated using scikit-learn [19].

\section{Results \& Discussion}

\section{The IEDB dataset represents large pathogen variety}

In order to achieve a bias-free prediction of epitopes, it is essential to have a large variety of known epitopes from evolutionarily distinct organisms in the training set. Therefore, we initially analyzed the taxonomic origin of the protein sequences provided by the IEDB and visualized the results with the online tool Pavian metagenomics data explorer [20], see Figure 3. Since the number of verified regions per protein varies, we analyzed them separately. Our filtered dataset contains 1,798 proteins with 24,610 epitopes and nonepitopes assigned to them.

At the protein level, the data consist of 16 different families. The four families of Bacteria account for $20.6 \%$, the five families of Eukaryota for $54.2 \%$, and the seven families of Viruses for $25.1 \%$ of all proteins in the training data. At the level of the verified regions, we observed a slight shift, so that Eukaryota with $61.0 \%$ are even more pronounced than the Bacteria with $15.7 \%$ and the Viruses with $23.4 \%$. Overall, the training data display a sufficient degree of taxonomic diversity. 
bioRxiv preprint doi: https://doi.org/10.1101/2020.05.12.090019; this version posted May 25, 2020. The copyright holder for this preprint (which was not certified by peer review) is the author/funder, who has granted bioRxiv a license to display the preprint in perpetuity. It is made available under aCC-BY-NC 4.0 International license.

\section{Proteins}
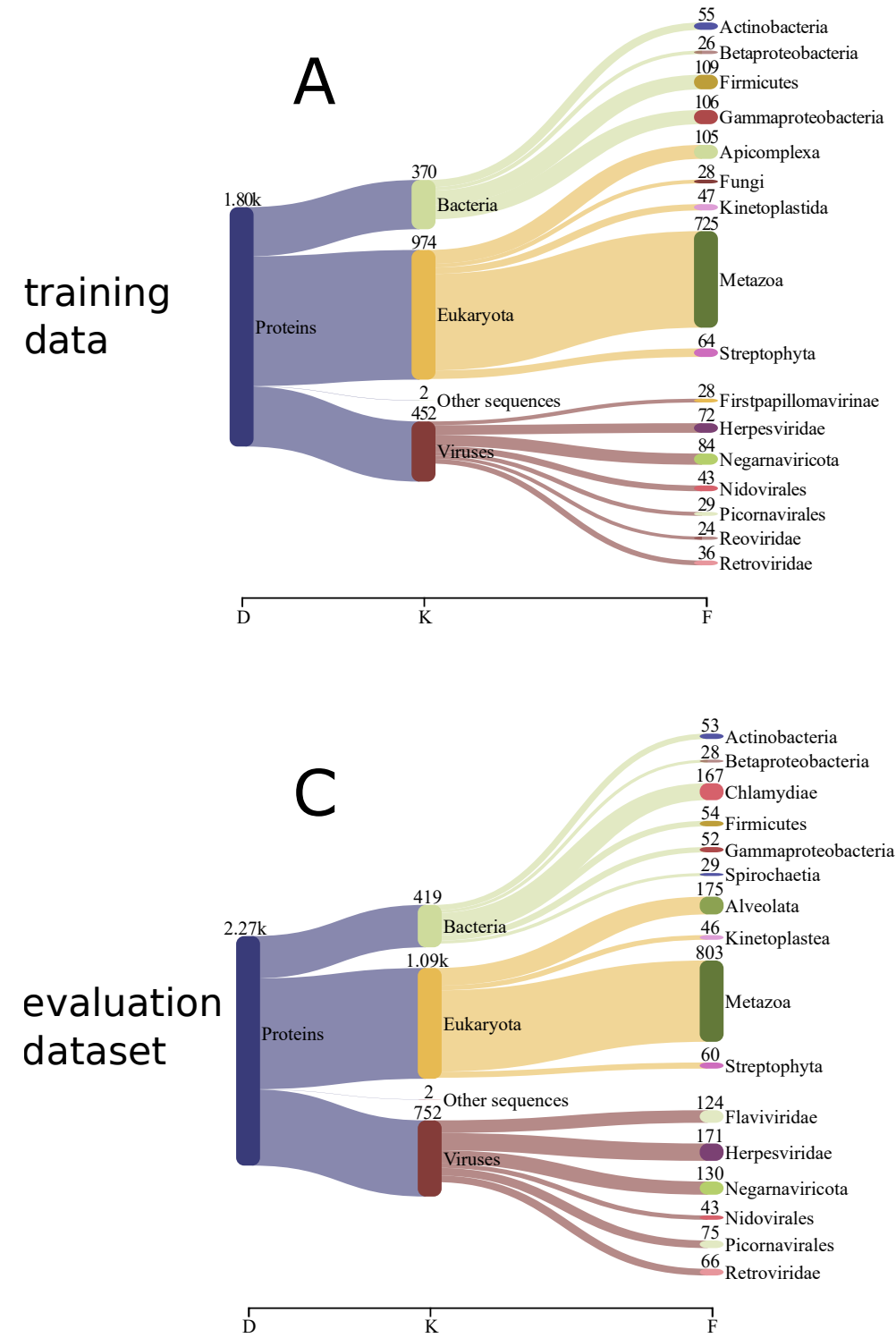

verified Regions
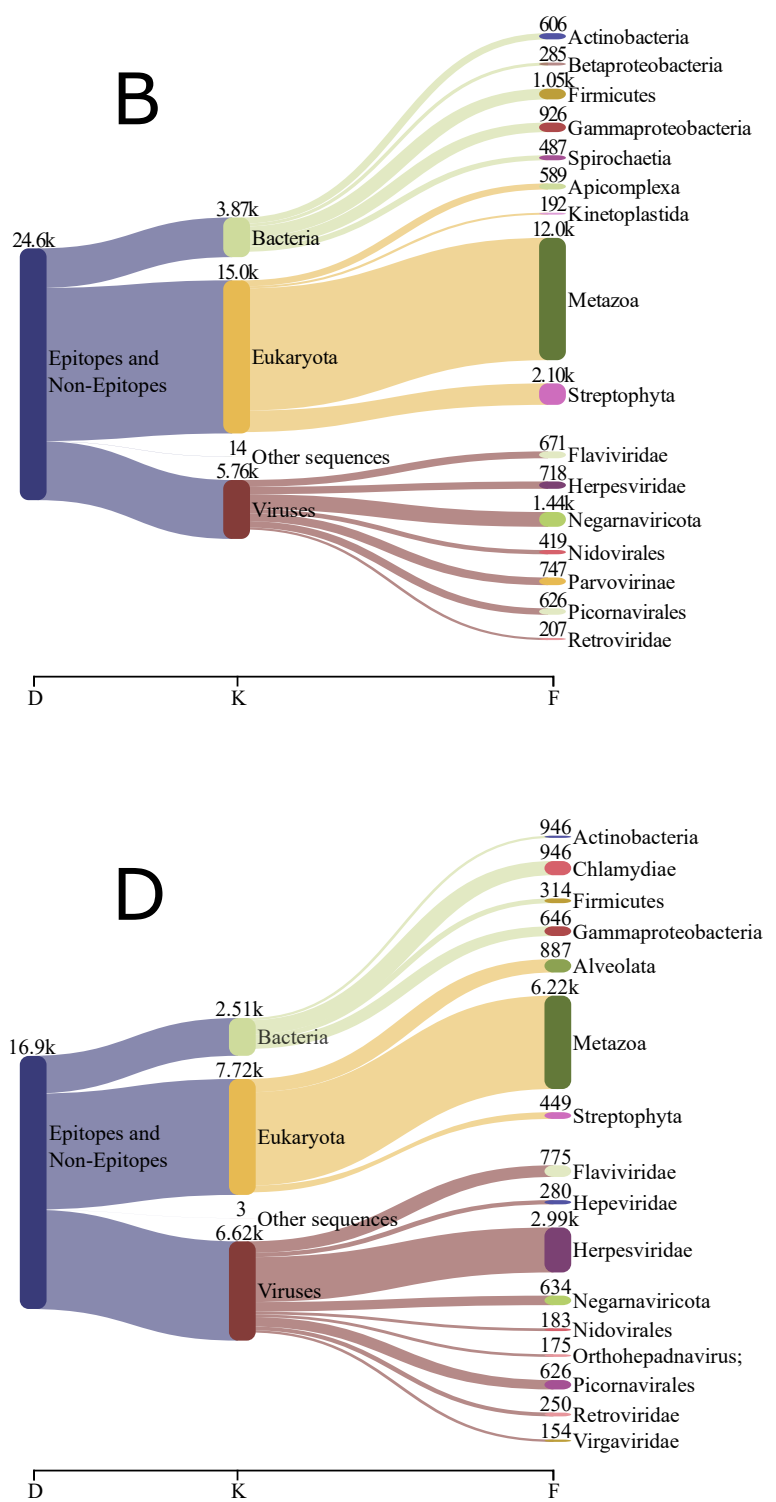

Figure 3: Taxonomic origin of the training data $(\mathrm{A}$ and $\mathrm{B})$ and evaluation dataset $(\mathrm{C}$ and $\mathrm{D})$. A shows the origin of all 1,798 proteins in the training data, covering a wide variety of kingdoms and families. B shows the taxonomic origin of the epitopes and non-epitopes, with $15.7 \%$ from Bacteria, $61 \%$ Eukaryota and $23.4 \%$ from Viruses. Taxonomic origin of the sequences used in the evaluation dataset: $\mathrm{C}$ shows the origin of all 2,267 proteins and D shows the taxonomic origin of the epitopes and non-epitopes, with $14.9 \%$ from Bacteria, $45.7 \%$ from Eukaryota and $39.2 \%$ from Viruses. 


\section{Evaluation of EpiDope via 10-fold cross-validation}

As described in the methods section, we evaluated the performance of EpiDope with competing methods on the training data, using 10-fold crossvalidation. Each of the ten subsets consists of proteins that have a sequence identity below 0.5 to all proteins in the other nine subsets (for details, see the 'Data preparation' section).

The Receiver operating characteristic (ROC)-curve (Figure 4A) shows that EpiDope is with an AUC of $0.67 \pm 0.07$ clearly outperforming competing prediction approaches, all of which achieved an $\mathrm{AUC} \leq 0.56$. Despite multiple requests from us to the developers of BepiPred2, we could not confirm their stated AUC performance of 0.57 on our reduced and less redundant dataset (see Table 1).

Usually, it is not necessary to find all immunodominant epitopes in a proteome. Instead, only a small number of functioning epitopes are required, ideally without having to scan a large number of regions. Therefore the prediction performance in the highest-rated regions is of particular interest. To evaluate the performance of these regions, we used AUC10\%. The AUC10\% is the AUC of the ROC curve for a False Positive Rate $(\mathrm{FPR})<0.1$, normalized, as suggested by the BepiPred2 developers by multiplying with 10 [9].

Table 2: Comparison of the AUC and AUC10\% of the ROC curve calculated for multiple tools, on the training data. AUC shows the value on the complete ROC curve while AUC10\% is the area for a False Positive Rate $(\mathrm{FPR})<0.1$ corrected by multiplying with 10. EpiDope is compared using 10 -fold cross validation.

\begin{tabular}{l|l|l}
\hline \multicolumn{3}{c}{ training data } \\
\hline Tool & AUC & AUC10\% \\
\hline EpiDope & 0.670 & 0.151 \\
Betaturn & 0.562 & 0.070 \\
Accessibility & 0.548 & 0.058 \\
Antigenicity & 0.443 & 0.034 \\
Hydrophilicity & 0.521 & 0.053 \\
IUPred & 0.489 & 0.060 \\
Bepipred2 & 0.490 & 0.038 \\
\hline random & 0.500 & 0.050
\end{tabular}

EpiDope reaches a high AUC10\% of 0.151, compared to the second best method (Betaturn) with an AUC10\% of 0.070 (see Table 2). Note that Jespersen et al. state an AUC10\% of 0.08 for BepiPred2 9] on the original unreduced dataset. The performance of EpiDope relies on the high precision of the top predictions, notable also in the Precision-Recall curve, see Figure $4 \mathrm{~B}$.

\section{Evaluation on new data}

We created a new dataset that contains all verified regions that were not included in the BepiPred2 dataset (as of 27.11.2019). All areas that were tested positive by at least two assays were stored as epitopes, whereas all areas tested in at least two assays and not tested positive in any assay were stored as non-epitopes. These are the same conditions that were used to create the BepiPred2 dataset [9]. Thus this dataset is entirely independent of our training dataset. This second dataset is from now on called evaluation dataset. The evaluation dataset was not pre-processed like the previous dataset (see Figure 1), to preserve a high amount of samples.

In comparison with the training data the evaluation dataset is of comparable size (see Figure $3 \mathrm{~B}$ and $3 \mathrm{D}$ ), with the training data having over 24,600 verified regions and the evaluation dataset having over 16,900 verified regions. The evaluation dataset has a lower proportion of eukaryotic verified regions $(45.7 \%)$ and a higher proportion of viral verified regions $(33.1 \%)$ compared the training data (eukaryotic $61.0 \%$, viral $23.4 \%$ ). From the 16 most common families (Figure 3D), five were not present in the training data. Theses families are Chlamydiae, Alveolata, Hepeviridae, Orthohepadnavirus, and Virgaviridae, representing $5.6 \%$, $5.25 \%, 1.66 \%, 1 \%$ and $0.91 \%$ of the evaluation dataset. The family of Herpesviridae is in the evaluation dataset over six times more common, with $17.75 \%$ as compared to the training dataset $(2.92 \%)$.

Furthermore, the ratio of epitope regions vs. nonepitope regions changed dramatically, from $35 \%$ epitopes in the training dataset to $58 \%$ in the evaluation dataset.

As with the training dataset, we calculated the predictions of several tools (see 'Evaluation of epitope prediction approaches' section for further details) for the verified regions. We calculated the ROC curve and the Precision-Recall curve (see Figure $4 \mathrm{C} / \mathrm{D})$ and evaluated the AUC and AUC10\% of EpiDope and the competing tools (see Table 3).

The ROC-curve (see Figure $4 \mathrm{C}$ ) shows that EpiDope is, with an AUC of 0.60, surpassing competing prediction approaches. We can observe a distinctly different result for nearly all tools in comparison with the evaluation on the training data. The AUC of EpiDope decreased by 0.065, which is within the calculated standard deviation of 0.071 . The analysis of the origin of the validation sites and 
bioRxiv preprint doi: https://doi.org/10.1101/2020.05.12.090019; this version posted May 25, 2020. The copyright holder for this preprint (which was not certified by peer review) is the author/funder, who has granted bioRxiv a license to display the preprint in perpetuity. It is made available under aCC-BY-NC 4.0 International license.
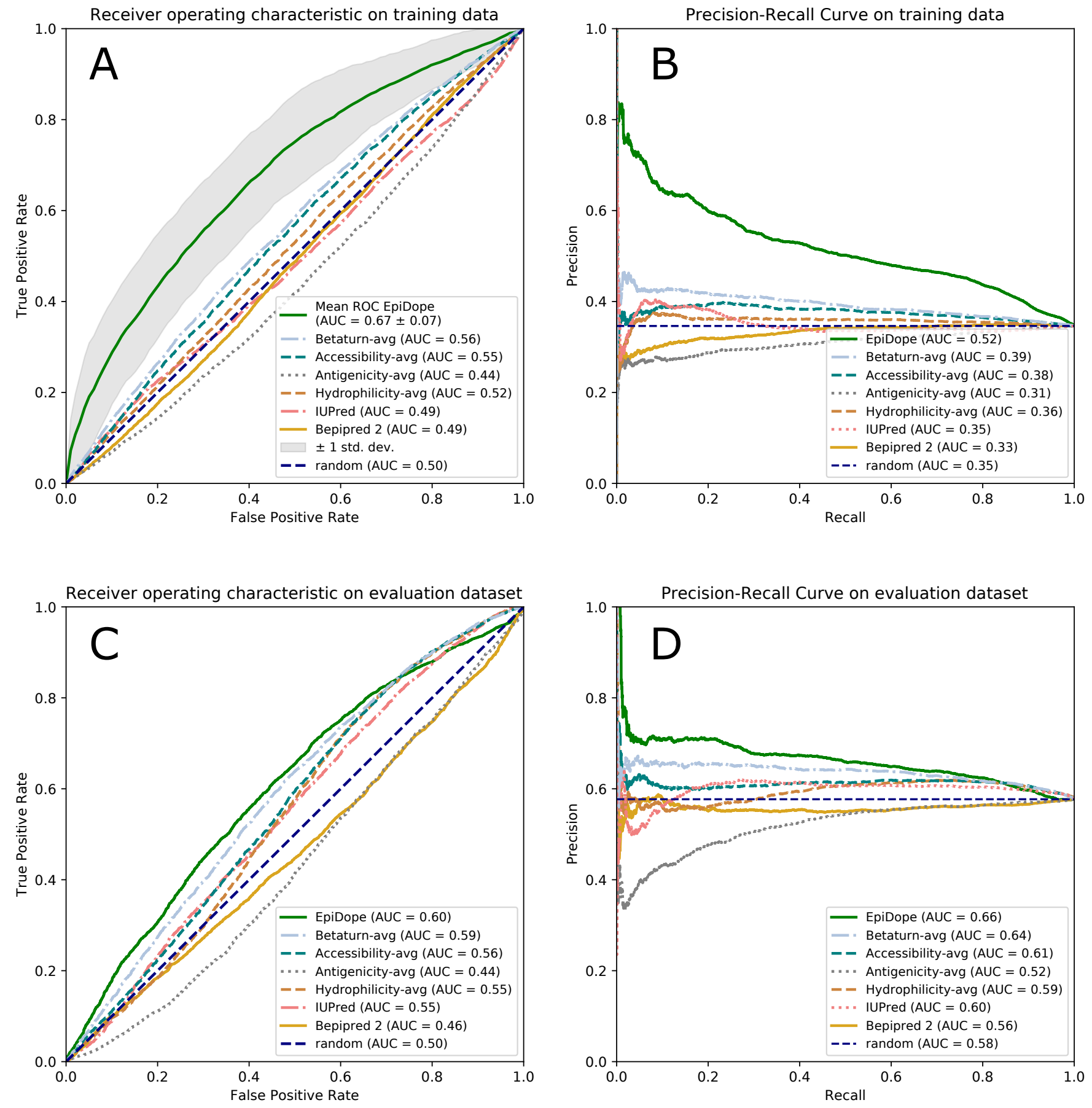

Figure 4: A shows the comparison of the ROC curves between the evaluated tools based on the training dataset. For the mean EpiDope ROC curve, every subset of the 10-fold cross-validation was predicted by the model that did not include this subset in its training data. This resulted in ten ROC curves for which the mean ROC curve was calculated (displayed in green) and the standard deviation area (grey). The other ROC curves were calculated on the same data without having to combine multiple predictions, as these tools and scores did not use this data for training. In $\mathrm{B}$, the precision-recall curve shows the trade-off between the number of false positive predictions compared to the number of false negative predictions. This is important as the number of epitopes and non-epitopes are not balanced in the dataset. C shows the ROC curves for the evaluation dataset, which consists of all currently (as of 27.11.2019) well verified regions that are not present in the training data. D shows the precision-recall curve on the evaluation dataset. 
proteins used in this evaluation dataset shows five new families, while at the same time the distribution according to kingdom changes. This reduces the similarity with the training dataset. A lower similarity with the training data leads to somewhat limited comparability with the evaluation on the training data. At the same time, however, it indicates how well the model has generalized the epitope prediction task to the more diverse data.

The AUC10\% indicates the usability of all methods for practical applications. EpiDope outperforms all competing methods with an AUC10\% of 0.089 (see Table 3). The second best method betaturn reaches an AUC10\% of 0.069 . These results indicate that EpiDope can predict even data that is relatively distinct from the training data and has a higher usability than competing methods.

Table 3: Comparison of the AUC and AUC10\% of the ROC curve calculated for multiple tools on the evaluation dataset. AUC shows the value on the complete ROC curve, while AUC10\% is the area for a false-positive rate $(\mathrm{FPR})<0.1$ corrected by multiplying with 10 .

\begin{tabular}{l|l|l}
\hline \multicolumn{2}{c}{ evaluation dataset } \\
\hline Tool & AUC & AUC10\% \\
\hline EpiDope & 0.605 & 0.089 \\
Betaturn & 0.589 & 0.069 \\
Accessibility & 0.563 & 0.058 \\
Antigenicity & 0.436 & 0.021 \\
Hydrophilicity & 0.550 & 0.048 \\
IUPred & 0.550 & 0.040 \\
Bepipred2 & 0.465 & 0.048 \\
\hline random & 0.500 & 0.050
\end{tabular}

\section{EpiDope output and visualization}

EpiDope produces multiple outputs. As an easily readable and interpretable format, EpiDope visualizes its results in an interactive html plot using the Python bokeh package [21]. This allows large proteins to be displayed without impairing readability. For an example output plot see Figure 5. By default, EpiDope highlights regions of at least eight consecutive amino acids that have predicted values above the threshold of 0.818 . This threshold corresponds to a $15 \%$ recall rate with a precision of 0.635 on the training data. Furthermore the user can provide amino acid sequences (text file with one sequence per line) that are either classified as epitopes or non-epitopes to highlight them in the html plot as blue or red regions, respectively.

Additionally, EpiDope produces simple computer parsable output. The file epidope_scores.csv lists the predicted score per amino acid, predicted_epitopes.csv lists all regions with a score higher than the defined threshold and predicted_epitopes_sliced.faa is a multi-fasta file of potential epitopes with a user defined size and overlap, that can be used to scan for epitopes in wet lab experiments.

\section{Conclusion}

In this study, we have developed the linear Bcell epitope prediction tool EpiDope (Epitope Deep learning predictor).

While only requiring a protein's amino acid sequence, EpiDope has been shown to be the bestperforming among currently available B-cell epitope prediction tools. EpiDope is based on a Deep Neural Network. We trained EpiDope on almost 25,000 experimentally verified epitope and non-epitope regions. We have used two different datasets to compare the performance of EpiDope with numerous different tools, including the currently probably most used tool, BepiPred2. For the training data, we performed 10-fold crossvalidation to ensure the reliability of the benchmarks. In addition, all proteins of a subset have a sequence identity of less than $50 \%$ to the proteins in the other nine subsets. This ensures that all ten subsets are independent of each other. The second dataset (evaluation dataset) consists of almost 17,000 new verified epitopes and nonepitopes, which therefore have not been present in the training dataset. On both datasets EpiDope outperformed all competing methods. Especially for the AUC10\%, which represents the performance on the practically relevant top predictions. The AUC10\% was corrected by multiplying with 10 (as described in BepiPred2 [9]) and varies between 0.151 and 0.089 .

The training data contains many short validated regions for which a high false-negative rate is expected [22]. Those false-negative cases result in a more conservative prediction for epitopes. However, the effect in real-world applications is minimal, as only a high true-positive and a low falsepositive rate are important to reduce the lab work. These cases are best represented by AUC10\%.

The high predictive power of EpiDope enables a much more precise search for epitopes and, thus, faster and more cost-effective development of medical treatments or diagnostic methods.

For even easier usability for a broad research com- 


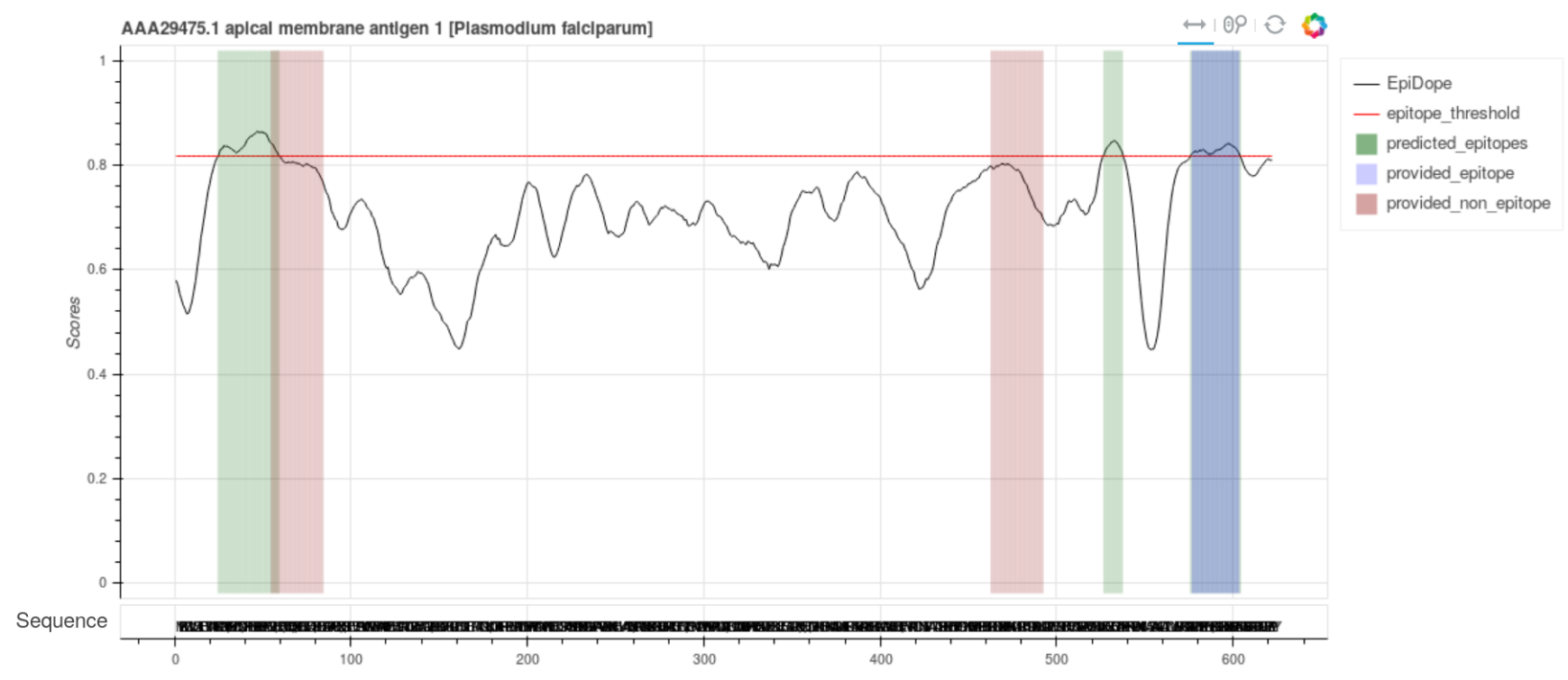

Figure 5: Graphical output of EpiDope as an interactive html plot for the apical membrane antigen 1 of Plasmodium falciparum. The black line displays the predicted values of EpiDope per position (amino acid). A higher value means that EpiDope certifies this region a higher chance of being an epitope. The red line is the default threshold for the predicted epitopes. The green regions are the predicted parts that are above the threshold for at least 8 consecutive amino acids. The blue and green regions are user provided regions that are known to be epitopes and non-epitopes, respectively.

munity, we plan to establish an EpiDope Online version. Until then EpiDope can be downloaded from GitHub (github.com/mcollatz/EpiDope)or installed via Conda. The training datasets are available in the open science framework (osf.io/krw2j).

\section{Competing Interests}

The authors declare no competing interests.

\section{Funding}

This work was funded in the framework of the national research network InfectControl, project "Molecular serology for rapid determination of vaccination titers (STIKO Serology)", which was financially supported by the Federal Ministry of Education and Research (BMBF) of Germany under grant 03ZZ0820A. The funders had no role in study design, data collection and analysis, decision to publish, or preparation of the manuscript.

\section{References}

[1] Rino Rappuoli, Mariagrazia Pizza, Giuseppe Del Giudice, and Ennio De Gregorio. Vaccines, new opportunities for a new society. Proceedings of the National Academy of Sciences, 111(34):12288-12293, 2014.
[2] Willem G Van Panhuis, John Grefenstette, Su Yon Jung, Nian Shong Chok, Anne Cross, Heather Eng, Bruce Y Lee, Vladimir Zadorozhny, Shawn Brown, Derek Cummings, et al. Contagious diseases in the united states from 1888 to the present. The New England journal of medicine, 369(22):2152, 2013.

[3] C Jessica E Metcalf, Jeremy Farrar, Felicity T Cutts, Nicole E Basta, Andrea L Graham, Justin Lessler, Neil M Ferguson, Donald S Burke, and Bryan T Grenfell. Use of serological surveys to generate key insights into the changing global landscape of infectious disease. The Lancet, 388(10045):728-730, 2016.

[4] Benjamin F Arnold, Heather M Scobie, Jeffrey W Priest, and Patrick J Lammie. Integrated serologic surveillance of population immunity and disease transmission. Emerging infectious diseases, 24(7):1188, 2018.

[5] Konrad Sachse, Kh Shamsur Rahman, Christiane Schnee, Elke Müller, Madlen Peisker, Thomas Schumacher, Evelyn Schubert, Anke Ruettger, Bernhard Kaltenboeck, and Ralf Ehricht. A novel synthetic peptide microarray assay detects chlamydia species-specific antibodies in animal and human sera. Scientific reports, 8(1):1-13, 2018.

[6] Marc HV Van Regenmortel. What is a b-cell epitope? In Epitope Mapping Protocols, pages 3-20. Springer, 2009.

[7] Jens Vindahl Kringelum, Morten Nielsen, Søren Berg Padkjær, and Ole Lund. Structural analysis of b-cell epitopes in antibody: protein complexes. Molecular immunology, 53(1-2):24-34, 2013. 
bioRxiv preprint doi: https://doi.org/10.1101/2020.05.12.090019; this version posted May 25, 2020. The copyright holder for this preprint (which was not certified by peer review) is the author/funder, who has granted bioRxiv a license to display the preprint in perpetuity. It is made available under aCC-BY-NC 4.0 International license.

[8] Jose L Sanchez-Trincado, Marta Gomez-Perosanz, and Pedro A Reche. Fundamentals and methods for T-and B-cell epitope prediction. Journal of immunology research, 2017, 2017.

[9] Martin Closter Jespersen, Bjoern Peters, Morten Nielsen, and Paolo Marcatili. Bepipred-2.0: improving sequence-based b-cell epitope prediction using conformational epitopes. Nucleic acids research, 45(W1):W24-W29, 2017.

[10] Yoshua Bengio. Deep learning of representations for unsupervised and transfer learning. In Proceedings of ICML workshop on unsupervised and transfer learning, pages 17-36, 2012.

[11] Weizhong Li and Adam Godzik. Cd-hit: a fast program for clustering and comparing large sets of protein or nucleotide sequences. Bioinformatics, 22(13):1658$1659,2006$.

[12] Michael Heinzinger, Ahmed Elnaggar, Yu Wang, Christian Dallago, Dmitrii Nachaev, Florian Matthes, and Burkhard Rost. Modeling the language of life-deep learning protein sequences. bioRxiv, page 614313, 2019.

[13] Sepp Hochreiter and Jürgen Schmidhuber. Long shortterm memory. Neural computation, 9(8):1735-1780, 1997.

[14] JMR Parker, D Guo, and RS Hodges. New hydrophilicity scale derived from high-performance liquid chromatography peptide retention data: correlation of predicted surface residues with antigenicity and x-rayderived accessible sites. Biochemistry, 25(19):54255432,1986

[15] Jean-Luc Pellequer, Eric Westhof, and Marc HV Van Regenmortel. Correlation between the location of antigenic sites and the prediction of turns in proteins. Immunology letters, 36(1):83-99, 1993.

[16] Emilio A Emini, JOSEPH V Hughes, DoS Perlow, and $\mathrm{J}$ Boger. Induction of hepatitis a virus-neutralizing antibody by a virus-specific synthetic peptide. Journal of virology, 55(3):836-839, 1985.

[17] AS Kolaskar and Prasad C Tongaonkar. A semiempirical method for prediction of antigenic determinants on protein antigens. FEBS letters, 276(1-2):172174,1990

[18] Zsuzsanna Dosztányi, Veronika Csizmok, Peter Tompa, and István Simon. Iupred: web server for the prediction of intrinsically unstructured regions of proteins based on estimated energy content. Bioinformatics, 21(16):3433-3434, 2005.

[19] F. Pedregosa, G. Varoquaux, A. Gramfort, V. Michel, B. Thirion, O. Grisel, M. Blondel, P. Prettenhofer, R. Weiss, V. Dubourg, J. Vanderplas, A. Passos, D. Cournapeau, M. Brucher, M. Perrot, and E. Duchesnay. Scikit-learn: Machine learning in Python. Journal of Machine Learning Research, 12:2825-2830, 2011.

[20] Florian P Breitwieser and Steven L Salzberg. Pavian: Interactive analysis of metagenomics data for microbiomics and pathogen identification. BioRxiv, page $084715,2016$.
[21] Bokeh Development Team. Bokeh: Python library for interactive visualization, 2019.

[22] Kh Shamsur Rahman, Erfan Ullah Chowdhury, Konrad Sachse, and Bernhard Kaltenboeck. Inadequate reference datasets biased toward short non-epitopes confound B-cell epitope prediction. Journal of Biological Chemistry, 291(28):14585-14599, 2016. 